\title{
Rheological Modeling of the Effects of Adulteration on Nigerian Honey
}

\author{
John Uju Nwalor1, Faith Uchenna Babalola1, Vincent Okechukwu Anidiobu ${ }^{2 *}$ \\ ${ }^{1}$ Department of Chemical Engineering University of Lagos, Lagos, Nigeria \\ ${ }^{2}$ Food Process Engineering Section, Department of Food Technology, The Federal Polytechnic, Ado-Ekiti, Nigeria \\ Email: *vinanidiobu@gmail.com
}

How to cite this paper: Nwalor, J.U., Babalola, F.U. and Anidiobu, V.O. (2018) Rheological Modeling of the Effects of Adulteration on Nigerian Honey. Open Journal of Fluid Dynamics, 8, 249-263. https://doi.org/10.4236/ojfd.2018.83016

Received: March 26, 2018

Accepted: July 6, 2018

Published: July 9, 2018

Copyright (c) 2018 by authors and Scientific Research Publishing Inc. This work is licensed under the Creative Commons Attribution-NonCommercial International License (CC BY-NC 4.0). http://creativecommons.org/licenses/by-nc/4.0/

\section{(c) (i) (8) Open Access}

\begin{abstract}
Honey was serially diluted with different percentages of glucose, fructose and water and each was analysed rheologically at room temperature of $27^{\circ} \mathrm{C}$. Pure honey exhibits thixotropic time-dependent rheological behaviour, the behaviour of glucose and fructose solutions in water (a Newtonian solvent) tends towards near Newtonian. The rheological profiles of pure and adulterated honey samples were determined using RV DV-III Ultra Programmable Rheometer at low rates of share. A Structural Kinetic Model was developed which provided good correlations with the rheological data. The new model was used to classify samples using their average molecular weights as one of the distinguishing parameters. Also the order of the kinetics in the new model suggests the number of active components in the "honey" undergoing deformation as 3. Carreau-Yasuda model was also improved upon to provide an independent assessment of average molecular weight of samples.
\end{abstract}

\section{Keywords}

Honey, Rheology, Structural Kinetic Model, Amended Carreau-Yasuda Model, Honey Adulteration

\section{Introduction}

Honey is a sweet fluid produced by honey bees (Apis Mellifera) from the nectar of flowers [1]. It is composed of mainly carbohydrates such as monosaccharides (glucose and fructose) and oligosaccharides like sucrose, maltose, melezitose, and raffinose. Pure honey also contains proteins, fats, water, vitamins and minerals. It is reputed to have a diverse set of nutritional and medicinal benefits. These and honey's pleasant taste of universal appeal ensure a sustained high demand for the product. The supply of honey is, however, quite constrained and 
hardly ever matches the demand. Consequently, the product always commands a relatively high price and provokes adulteration and imitation by fraudsters. Sugar based products such as fructose, glucose, sucrose, and starch are used by unscrupulous vendors to adulterate honey. Some bee keepers even feed their insects with the aforementioned to enhance the volume of "honey" produced and, hence, to maximize profit at the expense of the product's quality. Experienced fake honey vendors add honey flavour to caramels to mimic honey. Adulterated or fake honey would not be expected to have the potency of the genuine product as they would contain little or none of the constituents that impart nutritional and medicinal values to the latter. There is, thus, a strong imperative to constantly ascertain the quality of what is sold in the market as honey for public consumption, to hospitals for medicinal applications and to the industry for commercial purposes.

This need is presently met by means of time consuming and expensive procedures based on physicochemical characterizations of the product. In view of the wide and varied use to which honey is put and in view of the varied scale of its utilization, a simple and inexpensive characterization of the integrity of fluids passed off as honey is called for. This study explores the efficacy of a rheological characterization method to meet this need.

Rheology is the study of deformation and flow of fluids. The goal is to employ it to understand and predict the behaviour of fluids undergoing deformation. The rheological responses of fluid materials are obtained in terms of the dependence of their apparent viscosities on shear rate, and shear stress. A good understanding of rheology is essential to understanding many processes in engineering and other areas of research. All materials are made up of molecules under relative motion. These molecular motions are influenced by interaction potentials arising from the presence of the molecules. The same molecular motions and interactions are responsible for rheology [2].

There are many types of fluids in nature-pure substances, mixtures, dispersions and solutions, which fall into categories of either simple or structured fluids [3]. Structured fluids, unlike Newtonians, do not obey simple linear relationship between applied shear stress and shear rate [4]. Nearly all these materials have their viscosities decreasing with increasing rate of shear. This phenomenon of shear thinning becomes progressively larger as the volume concentration of oligomers increases. The observed rheological responses of fluids reflect their macroscopic state. They are, however, affected by the changes and properties at the molecular or microscopic constitution. A major challenge is to establish links between macroscopic rheological properties with changes at the molecular and microscopic levels [5]. Rheological data on honey together with data on its composition and structure should lead to understanding the relationship between them [6]. In turn such knowledge should lead to the improvement in the assessment of the integrity of honey presented for consumption through the development of cheap rheological methods. 
The objective of this study is firstly to explore the efficacy of utilizing honey rheology to assess its purity and to extract structural and compositional information from the theological data.

\section{Theory}

The essence of this section is to develop a structural kinetic model that can distinguish pure from adulterated honey using average molecular weight as one of its major distinguishing properties. Also, to improve upon Carreau-Yasuda model so that it can be used independently to assess the molecular weight of samples from the rheograms.

\subsection{Structural Kinetic Model for Honey}

The structural kinetic approach assumes that the change in the rheological behaviour is associated with shear-induced breakdown of internal structure of pure honey. We use the analogy of a chemical reaction to express the structural breakdown process in the following form:

$$
\text { Structured state } \rightarrow \text { Non Structured state }
$$

Honey is at rest at its structured state but undergoes deformation at its unstructured state. This model will assume in accordance with the above expression that the structure of honey changes under the effect of imposed shear but restores itself upon the withdrawal of shear.

This study suggests that pure honey exhibits thixotropic time dependent flow pattern. The structured state of the thixotropic structure at any time $t$ and under applied shear rate, $\gamma$, can be represented by a dimensionless structural parameter $(\theta)$ as given in Equation (2):

$$
\theta=\theta(\gamma, t)
$$

This was defined, according to [7], as:

$$
\theta(\gamma, t)=\frac{\eta-\eta_{\infty}}{\eta_{o}-\eta_{\infty}}
$$

where $\eta_{o}$, is the initial apparent viscosity at $t=0$ (structured state) and $\eta_{\infty}$, is the equilibrium apparent viscosity as $t \rightarrow \infty$ (non-structured state). Both $\eta_{o}$ and $\eta_{\infty}$ are functions of applied shear rate only. The dimensionless structured parameter, $\theta$ is subjected to the following conditions: at the fully structured state, $t=0$ and $\theta=\theta_{o}$, and at non-structured state, as $t \rightarrow \infty, \theta=\theta_{\infty}$.

The rate of structural breakdown can be expressed as:

$$
-\frac{\mathrm{d} \theta}{\mathrm{d} t}=k\left(\theta-\theta_{\infty}\right)^{n}
$$

where $k=k(\gamma)$ is the rate constant, and $\mathrm{n}$ is the order of the structural breakdown reaction.

Integrating Equation (4) between $t=t$ and $t=0$, we have:

$$
\int \frac{\mathrm{d} \theta}{\left(\theta-\theta_{\infty}\right)^{n}}=\int A^{o} \mathrm{~d} t
$$


which yields:

$$
\left(\theta-\theta_{\infty}\right)^{1-n}=t k(n-1)+C
$$

Solving for $C$ at and $\theta=\theta_{o}, t=t_{o}$ (initial value problem) we obtain:

$$
\left(\theta_{o}-\theta_{\infty}\right)^{1-n}=C
$$

Putting back $\mathrm{C}$ into Equation (6) at $t_{0}=0$ gives:

$$
\left(\theta-\theta_{\infty}\right)^{1-n}-\left(\theta_{o}-\theta_{\infty}\right)^{1-n}=\operatorname{tk}(n-1)
$$

Putting back Equation (3) into Equation (8)

$$
\text { gives: } \eta=\eta_{\infty}+\left(\eta_{o}-\eta_{\infty}\right)[t k(n-1)+1]^{1 / 1-n}
$$

Equation (9) is designated as the Structural Kinetic Model (SKM) which, in this study, would be applied to honey. This equation should answer the need to have a model that can give an insight into the composition of honey. One way to make this connection is through appropriate introduction of the average molecular weight of the honey sample. A good relationship has been found for polymeric materials in the Mark Houwink relation as given in Equation (10).

$$
\eta_{o}=K M^{A}
$$

where $\eta_{o}$ is the zero shear Viscosity of the fluid, $K$ and $A$, are Mark Houwink constants. $A$ is generally in the range of 0.5 to 0.8 [8] and $M$ is the molecular weight of the fluid. $\gamma$, is the shear rate of fluid, $\eta_{\infty}$ is the time shear viscosity, $n$, is the order of structural breakdown reaction which is the number of active components taking part in the deformation, $t$ is the minimum relaxation time to obtain consistent rheological results. $k$ is the rate of deformation of fluid.

Putting Equation (10) into Equation (9) gives:

$$
\eta=\eta_{\infty}+\left(K M^{A}-\eta_{\infty}\right)[k t(n-1)+1]^{1 / 1-n}
$$

Equation (11) is designated as SKM for molecular weight Correlation in Honeys.

\subsection{Modification of Carreau-Yasuda Model}

The Carreau-Yasuda model [9], an empirical model developed for polymeric fluids and found to give excellent fit to pure honey rheology, is improved upon in this section by inserting the Mark Houwink relation to enable it correlate the average molecular weight of honey from the rheological parameters. The Carreau-Yasuda model is given as:

$$
\eta(\dot{\gamma})=\eta_{\infty}+\left(\eta_{o}-\eta_{\infty}\right)\left[1+(\dot{\gamma} \lambda)^{a}\right]^{\frac{n-1}{a}}
$$

This empirical model has five adjustable parameters, $\alpha, \lambda, n, \eta_{o}$ and $\eta_{\infty}$.

This model describes non-Newtonian time dependent flow with asymptotic viscosities at zero $\left(\eta_{o}\right)$ and infinite $\left(\eta_{\infty}\right)$ shear rates, and with no yield stress. The parameter $\lambda$ is the viscous relaxation time. The viscous relaxation time de- 
fines the location of the transition from shear-thickening to shear-thinning behaviour. The $1 / \lambda$ is the critical shear rate at which viscosity begins to decrease. The power-law slope is $(n-1)$. The value of $\mathrm{n}$ (consistency index) changes with the composition of the fluid. The parameter, " $\alpha$ " is the dimensionless parameter (sometimes called "the Yasuda constant" since it is a parameter added to Carreau equation by Yasuda). It describes the transition region between $\eta_{o}$ and the power-law region and it is inversely related to the breath of the zone.

Putting Equation (10) into Equation (12) yields:

$$
\eta(\dot{\gamma})=\eta_{\infty}+\left(K M^{A}-\eta_{\infty}\right)\left[1+(\dot{\gamma} \lambda)^{a}\right]^{\frac{n-1}{a}}
$$

Equation (13) is the Amended Carreau-Yasuda model (ACYM). A comparison shall be made on the molecular weight obtained using this equation and that from SKM.

\section{Methods}

The experimental methods involve samples preparation, rheological characterization and curve fitting.

\subsection{Sample Preparation}

Sample A is pure honey or control sample harvested and processed for the purpose of this work. Glucose G1 (produced by dissolving three parts of glucose in one part of distilled water), fructose F1 (produced by dissolving three parts of fructose in one part of distilled water) and distilled water sample named $\mathrm{H}_{0}$ were used to serially adulterate the control sample A. Samples G2, G3, G4 and G5 are $10 \%, 50 \%, 70 \%$ and $90 \%$ adulteration with sample G1. Likewise, Samples F2, F3, F4 and F5 are $10 \%, 50 \%, 70 \%$ and $90 \%$ adulteration of pure honey with sample F1. Also Samples H1, H2, H3, H4, H5 and H6 are 5\%, 10\%, 15\%, 20\%, 30\% and $50 \%$ adulteration with water.

\subsection{Rheological Characterization}

The rheological tests of the samples were carried out using Brookfield RV DV-III Ultra Programmable Rheometer. The analyses were done at room temperature of $27^{\circ} \mathrm{C}$. The equipment thermometer was kept dipped into the fluid before the analyses began for temperature control. The DV III Program mode was used. The shear rates, from 0.0 to $3.4 \mathrm{~s}^{-1}$ at incremental steps of $0.01 \mathrm{~s}^{-1}$ were used. The time interval for each speed was sixty seconds, and readings were taken every ten seconds. Step time was not allowed in order not to allow the recovery of the sample from the shearing of the initial speed. The samples were allowed to stand in the container for at least 30 minutes before the analysis to allow for the complete structural equilibrium. Coaxial spindle was chosen which is appropriate for the rheology of viscous non-Newtonian fluids. The geometries of coaxial cylinder are needed for application where extremely well-defined shear rate and shear stress are required. The spindle number " 00 " was chosen which 
would enable the Rheometer to calculate the shear stress $\left(\mathrm{N} / \mathrm{m}^{2}\right)$, viscosity (mPa.s), and torque (\%) for every shear rate $\left(\mathrm{s}^{-1}\right)$.The experimental procedure was repeated after 24 hours to determine if the results were reproducible.

\subsection{Rheological Curve Fitting}

For the Carreau-Yasuda model, curve fitting was carried out using the method of Morrison [10]. For the structural kinetic model, time resolved data on fluids' apparent viscosities at given constant rates of shear were extrapolated to zero times to obtain the zero shear viscosities. The zero shear (zero time) viscosity was applied in Mark-Houwink equation to obtain the fluid's molecular weight at each shear rate. The average molecular weight was then determined for all shear rates studied. Since this work was done at low rates of shear, the fluid might not have deformed long enough to give values of the infinite time viscosity for the calculation of rate of deformation, $k$. From Equation (9), the rate of deformation $(k)$ is obtained explicitly from:

$$
\begin{gathered}
k=\left\{\left[\left(\eta-\eta_{\infty}\right) /\left(\eta_{o}-\eta_{\infty}\right)\right]^{1-n}-1\right\} / t(n-1) \\
\text { Also, if } \Gamma \text { is defined by, } \Gamma=\left(\frac{\eta-\eta_{\infty}}{\eta_{o}-\eta_{\infty}}\right)^{1-n} \\
\text { Then } \Gamma=t k(n-1)+1
\end{gathered}
$$

Since $k$ is expected to depend only on rate of shear rate [7], a scheme was developed by which an optimized value of $k$ was determined for each rate of shear. The scheme associates optimum $k$ with the set of parameters that yielded the least coefficient of variation. This coefficient of variation or relative standard deviation (RSD) is the standard deviation of $k$ divided by its mean value calculated from the six different times of the experimental determination of apparent viscosities ( $10 \mathrm{~s}, 20 \mathrm{~s}, 30 \mathrm{~s}, 40 \mathrm{~s}, 50 \mathrm{~s}$ and $60 \mathrm{~s}$ ). This value of $k$ and the set of the other parameters that produced it are deemed as the parameters of the model and were found to also give the best fit, following Equation (16), to $\Gamma$ versus $t$ data.

\section{Results and Discussion}

This study focused on the effect of fructose, glucose and water adulteration on the rheological behaviour of honey. The ACYM and the new Structural Kinetic Model were used in the study. The rheological characterization of polymers and polymers in dilute solutions are well established [11]. It could be adopted to characterize honey since it contains some oligomers. It has been reported that honey contains as high as $11 \%$ melezitose which is an oligomer [12] [13]. This $11 \%$ percent concentration of melezitose in honey is to our advantage high since polymers as low as $2 \%$ concentration in solution exhibits non-Newtonian behaviours [11] [14].

Pure honey first exhibited shear thickening behaviour at the inception of flow 
and at low shear rate but later assumed an essentially shear thinning behaviour at higher rate of shear. Kurzberck and coworkers, [15] suggested that the presence of chain branches gave rise to strain hardening which they said was a necessary property of stability for polymers undergoing deformation (Figures 1-3). The clusters and aggregates of particles originally present as colloids or suspensions in the fluid were reversibly destroyed by shear. Triantafillopoulos, [16], explained this time-dependent shear thinning behaviour to be as a result of isothermal and reversible disorientation of the structures of the fluid. Venugopal and Abhilash [17], studied the hydration kinetics and rheological behaviour of guar gum which is a polymer. They inferred that decrease in viscosity with an

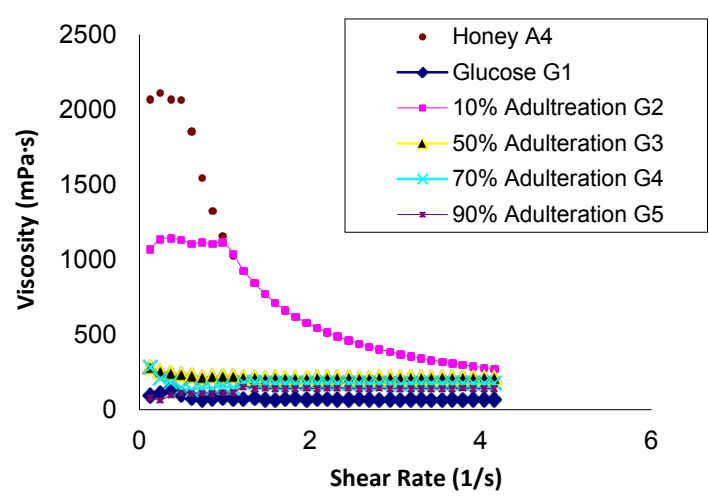

Figure 1. Effect of glucose adulteration on honey rheology at $27^{\circ} \mathrm{C}$.

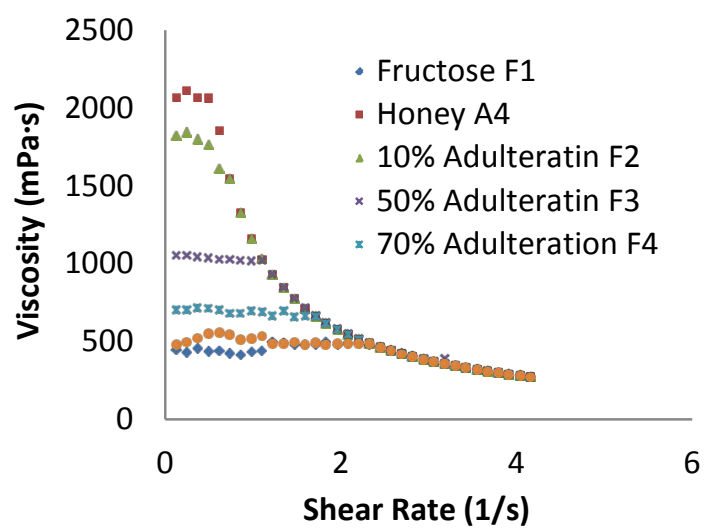

Figure 2. Effect of fructose adulteration on honey rheology at $27^{\circ} \mathrm{C}$.

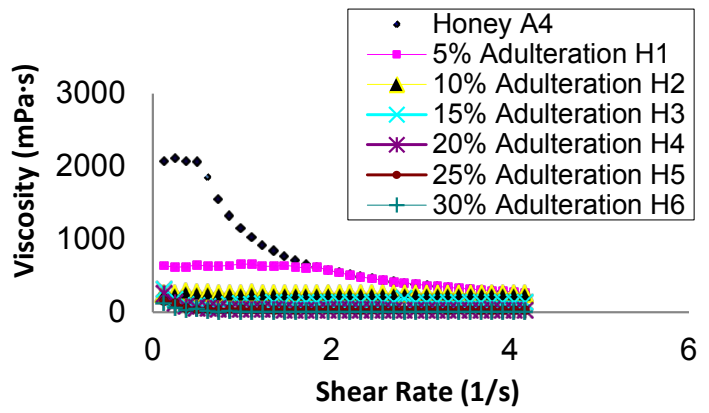

Figure 3. Effect of water adulteration on honey rheology at $27^{\circ} \mathrm{C}$. 
increase in shear rate over time was caused by inducing changes in the network entanglement of the fluid. This kind of flow behaviour was also reported by Fan and coworkers, [18] who worked on Numerical simulation of pulsatile non-Newtonian flow in the Carotid artery bifurcation. They suggested that at very low shear rate, similar to those employed in this work, blood exhibits shear thinning behaviour, but as shear rate increases, the non-Newtonian behaviour gradually diminishes. Stelmakeiene and coworkers, [19], Cohen and Weihs, [20], Mossel and coworkers, [21] and Munro, [22], all reported that honey exhibited thixotropic rheological behaviour. However, this is contrary to Zaitoun and coworkers [23], who concluded that honey exhibits Newtonian behaviour. It may be concluded that care must be taken in choosing the rates of share range for a rheological characterization of honey intended for quality assessment. The foregoing observation influenced the study's choice of rheometer settings in this work for the rheological profiling conducted.

Figure 1 shows the effect of glucose adulteration on the rheology of honey. A look at the rheograms indicates that the apparent viscosity of honey and its non-Newtonian feature decreases sharply with the addition of glucose. The more the adulterants are added, the more marked this effect until about $70 \%$ adulteration when the viscosity turns Newtonian. Figure 2 shows the effect of fructose adulteration on the rheology of honey. In this case there is a gradual decrease in the apparent viscosity of resulting sample with increasing adulteration. The gradual decreases suggest that there is more fructose than glucose in honey which was confirmed in the study of proximate anises of honey [24]. It has been suggested that the colloidal materials, polymerized monosaccharides, melezitose, raffinose and some oligosaccharides are responsible for honey's non-Newtonian behaviour [13]. The substitution of these heavy or high molecular weight materials with the lower molecular weight adulterants lead to sequential decrease in the viscosity of the resulting adulterated fluids.

Figure 3 shows the effect of water adulteration on the rheology of honey. Water is not a good candidate for honey adulteration as it shows in the physical appearance of the resulting fluid as early as $5 \%$ adulteration. From the rheograms (Figure 3), it can be observed that honey turns completely Newtonian from $10 \%$ adulteration with water. Other studies also show that adulterating honey with water leads to fermentation of the monosaccharides in honey leading to sour taste [19]. For better understanding of the rheograms, curve fits were carried out using both the newly derived Structural Kinetic model and the amended Carreau Yasuda model. The major difference between the two models, apart from the differences in the model parameters is that the new Structural Kinetic Model is a derived model while the modified Carreau-Yasuda is an empirical one.

Figure 4 shows the viscosity-time rheogram of sample A, used to obtain the zero shear viscosity at a shear rate of $0.01 \mathrm{~s}^{-1}$. The zero shear viscosity was applied in Mark-Houwink Equation (Equation (10)) to obtain the molecular weight at that shear rate. The average molecular weight of the fluid was then 


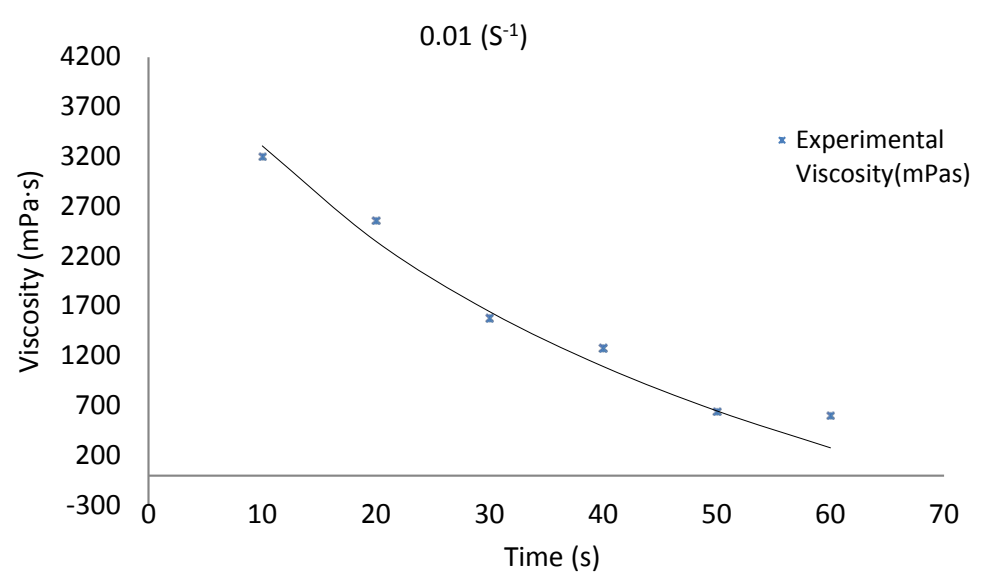

Figure 4. Viscosity-time rheogram of sample A at $27^{\circ} \mathrm{C}$.

determined for all the rates of shear used. Since this study was done at low shear rate, the fluid would not have deformed for a long time to obtain the infinite time viscosity. Figure 5 is the plot for optimization of infinite time viscosity at a shear rate of $0.01 \mathrm{~s}^{-1}$. From the plot, the minimum value (best value) of infinite time viscosity was obtained. It was observed during the iterations that the infinite (time) shear viscosities at different rates of shear that satisfied the SKM for pure honey were negative. This shall be discussed in details under Table 1. The determined optimum infinite time viscosity with the model parameters that produced it gave explicit estimates of $k$ from Equation (14). The mean value of $k$ for the constant rate of share data gave, as expected, the same value as was determined from the slope of the best linear fit to the data of Figure 6. Figure 7 shows the dependence of rate of deformation for sample A on rate of shear. The above sequence was repeated for all the samples used in this study.

The parameters of the structural kinetic model, $M$, is the molecular weight of honey, the Mark Houwink constants, $K$ and $A$, introduced into the equation via the Mark Houwink equation, the infinite time viscosity, $\eta_{\infty}$, which gives an indication of the viscosity of honey at knife edge (high shear) conditions, the zero time viscosity, $\eta_{0}$, and $n$, the order of structural breakdown kinetics which depends on the composition of the fluid.

Table 1 is the summary of rheological curve fitting results using the Structural Kinetic Model. It was observed that pure sample A, has its molecular weight as $252 \mathrm{~g} / \mathrm{mol}$. There are sequential decreases in the average molecular weight of adulterated honey with increases in adulteration. This is expected because the molecular weights of the adulterating materials are lower than that of pure honey. The high molecular weight values $(>180 \mathrm{~g} / \mathrm{mol})$ exhibited by pure samples in Table 1 and Table 2 could be as a result of high melezitose, colloidal materials, polymerized and crystalised monosaccharides in honey. The addition of glucose, which has a molecular weight of $180 \mathrm{~g} / \mathrm{mol}$, fructose which is an isomer of glucose with the same molecular weight and water with a molecular weight of $18 \mathrm{~g} / \mathrm{mol}$ lead to sequential decrease in the molecular weight of the resulting adulterated honeys. This could be attributed to the fact that all the materials 


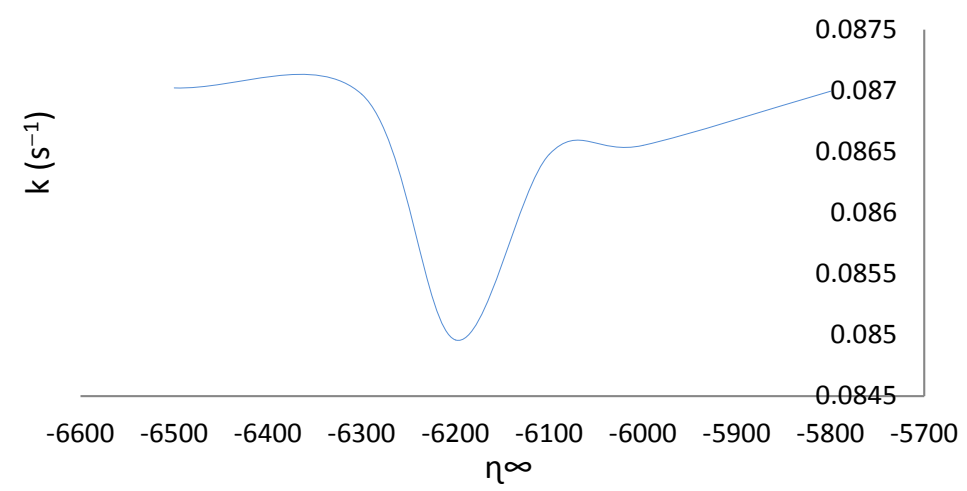

Figure 5. Optimisation of $\eta_{\infty}$ for Sample A at shear rate of $0.01\left(\mathrm{~s}^{-1}\right)$.

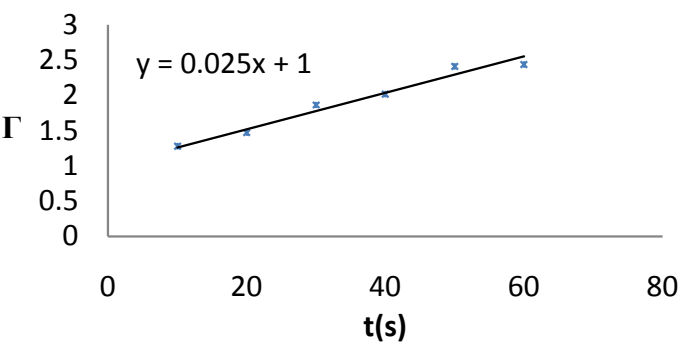

Figure 6. Plot of $\Gamma$ (from Equation (16)) against $\mathrm{t}$ at shear rate of $0.01 \mathrm{~s}^{-1}$.

Table 1. Summary of rheological curve fitting using our new Structural Kinetic Model (SKM).

\begin{tabular}{|c|c|c|c|c|c|c|}
\hline $\mathrm{S} / \mathrm{N}$ & Sample Code & Sample Identity & $\begin{array}{c}\text { Molecular } \\
\text { Weight }(\mathrm{g} / \mathrm{mol})\end{array}$ & $\mathbf{n}$ & $k\left(\mathrm{~S}^{-1}\right)$ & $t(s)$ \\
\hline 1. & A & Ijebu Mushin & 252.07 & 3.00 & 0.83 & 4.16 \\
\hline 2. & G1 & Glucose & 145.60 & 1.50 & 3.69 & 1.70 \\
\hline 3. & G2 & $10 \%$ Glucose Adulteration & 232.05 & 2.50 & 10.69 & 0.05 \\
\hline 4. & G3 & $50 \%$ Glucose Adulteration & 189.25 & 2.00 & 13.16 & 5.56 \\
\hline 5. & G4 & $70 \%$ Glucose Adulteration & 174.19 & 2.00 & 2.83 & 18.03 \\
\hline 6. & G5 & $90 \%$ Glucose Adulteration & 160.19 & 1.50 & 3.29 & 391.25 \\
\hline 7. & $\mathrm{~F} 1$ & Fructose & 148.60 & 1.50 & 3.26 & 1071.14 \\
\hline 8. & $\mathrm{~F} 2$ & $10 \%$ Fructose Adulteration & 230.36 & 2.50 & 4.12 & 0.19 \\
\hline 9. & F3 & $50 \%$ Fructose Adulteration & 198.25 & 2.50 & 5.18 & 0.0005 \\
\hline 10. & $\mathrm{~F} 4$ & $70 \%$ Fructose Adulteration & 176.19 & 2.00 & 1.59 & 30.00 \\
\hline 11. & F5 & $90 \%$ Fructose Adulteration & 160.13 & 1.50 & 4.06 & 17.49 \\
\hline 12. & $\mathrm{H} 1$ & 5\% Water Adulteration & 241.71 & 2.50 & 1.05 & 0.00003 \\
\hline 13. & $\mathrm{H} 2$ & $10 \%$ Water Adulteration & 222.20 & 2.00 & 0.94 & 0.42 \\
\hline 14. & $\mathrm{H} 3$ & 15\% Water Adulteration & 212.15 & 1.50 & 0.61 & 22.66 \\
\hline 15. & $\mathrm{H} 4$ & $20 \%$ Water Adulteration & 200.91 & 1.50 & 0.63 & 16.24 \\
\hline 16. & $\mathrm{H} 5$ & $30 \%$ Water Adulteration & 178.42 & 1.50 & 0.69 & 8.60 \\
\hline 17. & H6 & $50 \%$ water Adulteration & 132.44 & 1.50 & 0.75 & 5.31 \\
\hline
\end{tabular}




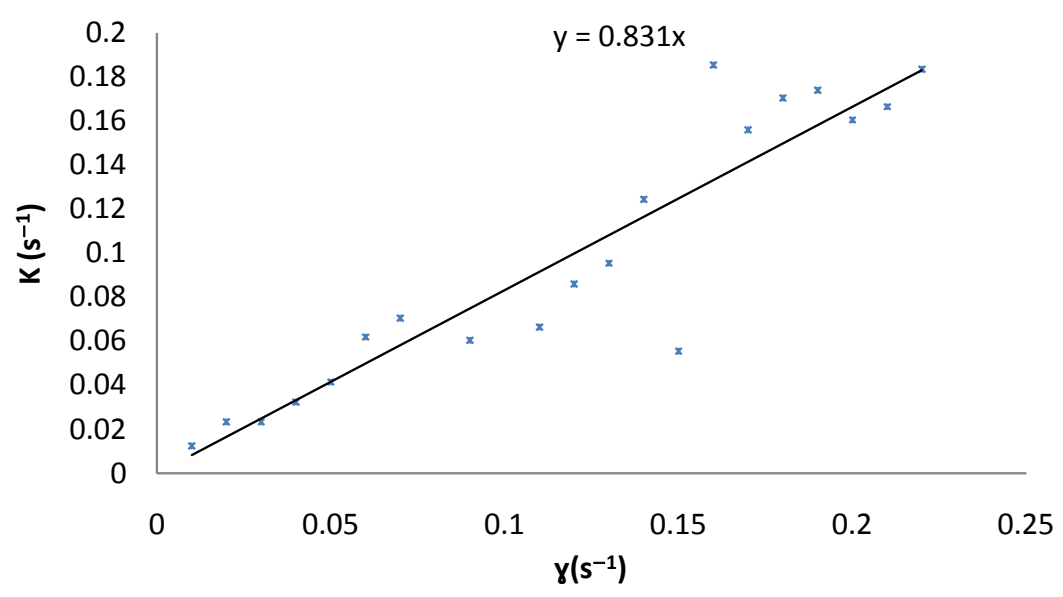

Figure 7. Plot of k against shear rate of sample A.

Table 2. Summary of rheological curve fitting using the Amended Carreau Yasuda Model (ACYM).

\begin{tabular}{|c|c|c|c|c|c|}
\hline S/N & Sample Code & Sample Identity & Molecular Weight & $\lambda$ & $\mathbf{n}$ \\
\hline 1. & A & Ijebu Mushin & 250.00 & 1.600 & -0.0013 \\
\hline 2. & G1 & Glucose & 147.00 & 0.887 & 0.9000 \\
\hline 3. & G2 & $10 \%$ Glucose Adulteration & 243.88 & 1.297 & 0.3700 \\
\hline 4. & G3 & 50\% Glucose Adulteration & 214.00 & 2368.94 & 0.5600 \\
\hline 5. & G4 & 70\% Glucose Adulteration & 200.88 & 11.48 & 0.8000 \\
\hline 6. & G5 & 90\% Glucose Adulteration & 187.00 & 43.37 & 0.9447 \\
\hline 7. & $\mathrm{~F} 1$ & Fructose & 149.81 & 13.08 & 0.9967 \\
\hline 8. & $\mathrm{~F} 2$ & $10 \%$ Fructose Adulteration & 241.27 & 1.51 & 0.5532 \\
\hline 9. & F3 & 50\% Fructose Adulteration & 216.91 & 0.85 & 0.7948 \\
\hline 10. & $\mathrm{~F} 4$ & $70 \%$ Fructose Adulteration & 200.21 & 0.60 & 0.8787 \\
\hline 11. & F5 & 90\% Fructose Adulteration & 187.00 & 0.005 & 0.8884 \\
\hline 12. & $\mathrm{H} 1$ & $5 \%$ Water Adulteration & 239.06 & 0.55 & 0.8494 \\
\hline 13. & $\mathrm{H} 2$ & $10 \%$ Water Adulteration & 226.68 & 2.15 & 0.9908 \\
\hline 14. & $\mathrm{H} 3$ & $15 \%$ Water Adulteration & 215.20 & 0.06 & 1.0078 \\
\hline 15. & $\mathrm{H} 4$ & $20 \%$ Water Adulteration & 200.12 & 0.06 & 0.5107 \\
\hline 16. & H5 & $30 \%$ Water Adulteration & 169.08 & 0.06 & -1.6938 \\
\hline 17. & H6 & $50 \%$ water Adulteration & 130.00 & 0.06 & 0.1418 \\
\hline
\end{tabular}

used in the adulteration here have lower molecular weight than honey and reductions in the molecular weight of adulterated samples explain why their behaviour tends toward Newtonian.

The best results were obtained for the pure honey samples at third order deformation kinetics. The essence of this is that three components of the honey constituents are active during deformation. It suggests that melezitose and other oligosaccharides and polymerized materials serve as the first, water as the 
second, and monosaccharides as the third active component. In the study of effect of water adulteration in honey, the results of curve fitting were best at second order deformation. It again suggests that the kinetic model views the entire honey as one component and the water as another during deformation.

The results of the rate of deformation of samples in Table 1 suggest that it increases with the adulteration of samples. It was observed during the iterations that the infinite (time) shear viscosities at different rates of shear that satisfied the SKM for pure honey were negative (Figure 5). Bacr and coworkers [25], provided the very first evidence of negative viscosity of magnetic fluids. It suggests that when shear is applied, the fluid behaves in a negative manner (having negative infinite shear viscosity) as a result of stored up energy due to the shear history of the fluid (Figure 5). This could be an expression of time dependent behaviour of honey. A similar behaviour was also observed by [26] in their study of Cooee bitumen. Earlier, [5] expressed frustration on the "very low magnitude" of infinite time viscosities of food products and consequently suggest that it should be ignored. It was worthy to note that the adulterated samples all gave positive infinite time viscosities. It then suggests that honey when adulterated assumed less time dependent rheological behaviour.

Figure 8 is the curve fit of honey rheogram using ACYM. Table 2 shows the summary of curve fitting results using the ACYM. The results show that pure honey sample A has an average molecular weight of $250 \mathrm{~g} / \mathrm{mol}$. This result is similar and close to the average molecular weight of $252.07 \mathrm{~g} / \mathrm{mol}$ obtained using SKM. Similarly, the molecular weight of samples decreases with increasing adulteration.

Figure 9 is the hysteresis loop observed on sample A's apparent viscosities when the shear rate trajectory was reversed in the opposite direction. A loop was observed between the upper and the lower ramps. The loop suggests that the fluid flows at lower energy level when the shear rate was reversed. This can only be true for fluids that remember its shear history. The loop quantifies the power loss during continuous input of energy into the fluid sample undergoing testing and it is independent of any thermal effects. It then suggests that honey exhibits thixotropic time dependent flow pattern. Thixotropy can be expressed as an isothermal, reversible reduction in apparent viscosities with shear rate. The amount of thixotropic breakdown is sensitive to previous shear history of honey. For time independent fluids, the curves would have been superimposed.

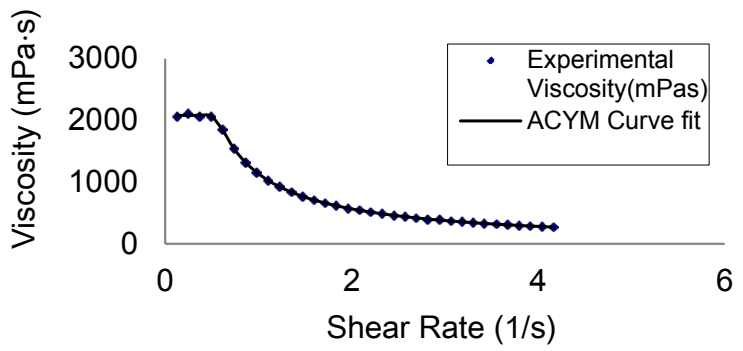

Figure 8. Amended Carreau Yasuda model fit of pure honey at $27^{\circ} \mathrm{C}$. 


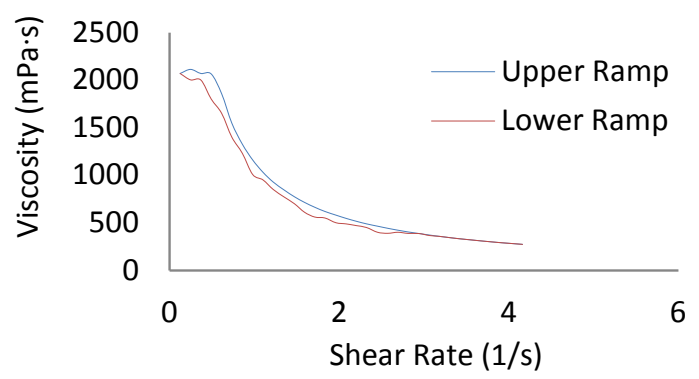

Figure 9. Hysteresis loop of pure honey at $27^{\circ} \mathrm{C}$.

\section{Conclusion}

Rheology is a consistent tool for honey characterization deriving from the correlation between flow behaviour and compositional changes in honey. Honey exhibited thixotropic rheological behaviour, glucose and fructose exhibited near Newtonian behaviour while water is Newtonian. The adulteration of honey with glucose, fructose or water pushes their viscosities towards Newtonian behaviour. The new Structural Kinetic Model followed well the experimental results and predicted the molecular weight of different samples as a means of discrimination of pure from adulterated honey and served to determine the degree of adulteration. The Carreau Yasuda model was amended to improve its performance as a tool for the determination of molecular weight of fluids from the rheological data. The amended Carreau Yasuda model compared relatively well with our new model except on honey adulterated with water where the empirical model failed.

\section{Acknowledgements}

We wish to thank the Tertiary Education Trust Fund (TETFUND) of Nigeria for sponsoring, at the University of Lagos, the Ph.D. program in Chemical Engineering that produced this article.

\section{References}

[1] White Jr., J.W. (1992) Quality Evaluation of Honey: Role of HMF and Diastase Assays. American Bee Journal, 132, 737-794.

[2] Bird, R.B, Stewart, W.E. and Lightfoot, E.N. (2002) Transport Phenomena. John Wiley \& Sons, Inc., New York, 1-251. https://doi.org/10.1115/1.1424298

[3] Franck, A.J. (2004) Understanding Rheology of Structured Fluids. An Instrument Publication.

http://www.tainstruments.com/pdf/literature/AAN016_V1_U_StructFluids.pdf

[4] Anidiobu, V.O. (2014) Rheological Modeling of the Effects of Sucrose Adulterant on Nigerian Honey. Nigerian Food Journal, 32, 103-112.

https://doi.org/10.1016/S0189-7241(15)30125-9

[5] Rao, M.A. (2006) Influence of Food Microstructure on Food Rheology, in Understanding and Controlling the Microstructure of Complex Foods. In: McClements, D.J., Ed., Woodhead Publishing Ltd., Cambridge.

[6] Nwalor, J.U., Babalola, F.U. and Anidiobu, V.O. (2014) Rheological Characterization of Honey: Application as an Index of Quality. In: Scales, P. and Ranganthan, P., 
Eds., Abstract Proceedings of the $6^{\text {th }}$ Pacific Rim Conference on Rheology, Melbourne, 211-212.

[7] Abu-Jdayil, B. (2003) Modeling the Time-Dependent Rheological Behavior of Semisolid Foodstuffs. Journal of Food Engineering, 57, 97-102.

https://doi.org/10.1016/S0260-8774(02)00277-7

[8] Launay, B., Doublier, J.R. and Cuvelier, G. (1986) Flow Properties of Aqueous Solutions and Dispersions of Polysaccharides. In: Mitchell, J.R. and Ledward, D.A., Eds., Functional Properties of Food Macromolecules, Elsevier Applied Science, London, $1-78$.

[9] Yasuda, K. (1979) Investigation of the Analogies between Viscometric and Linear Viscoelastic Properties of Polystyrene Fluids. Ph.D. Thesis, Massachusetts Institute of Technology, Cambridge.

[10] Morrison, F.A. (1999) Using the Solver Add-In in Microsoft Excel. http://pages.mtu.edu/ fmorriso/cm4650/Using_Solver_in_Excel.pdf

[11] Sunthar, P. (2008) Polymer Rheology. Department of Chemical Engineering, Indian Institute of Technology (IIT) Bombay, Mumbai. http://www.physics.iitm.ac.in/ compflu/Lect-notes/sunthar.pdf

[12] Lazaridou, A., Biliaderis, C.G., Bacandritsos, N. and Sabatini, A.G. (2004) Composition, Thermal and Rheological Behaviour of Selected Greek Honeys. Journal of Food Engineering, 64, 9-21. https://doi.org/10.1016/j.jfoodeng.2003.09.007

[13] Sopade, P.A., Halley, P.J., D’Arcy, B.R., Bhandari, B. and Caffin, N. (2004) Dynamic and Steady-State Rheology of Australian Honeys at Subzero Temperatures. Journal of Food Process Engineering, No. 27, 284-309. https://doi.org/10.1111/j.1745-4530.2004.00468.x

[14] De Laney, D. and Reilly, F. (2000) A New Approach to Polymer Rheology for Process and Quality Control Kayeness Instruments. https://zh.scribd.com/document/168083609/Dynisco-Practical-Rheology-Handboo $\underline{\mathrm{k}}$

[15] Kurzbeck, S., Oster, F., Munstedt, H., Nguyen, T.Q. and Gensler, R. (1999) Rheological Properties of Two Polypropylenes with Different Molecular Structure. Journal of Rheology, 43, 359-374. https://doi.org/10.1122/1.551040

[16] Triantafillopoulos, N. (1988) Measurement of Fluid Rheology and Interpretation of Rheograms. Kaltech Scientific, Inc., Novi, MI. http://ww.kaltecsci.com/rheology.pdf

[17] Venugopal, K.N. and Abhilash, M. (2010) Study of Hydration Kinetics and Rheological Behaviour of Guar Gum. International Journal of Pharma Science and Research, 1, 28-39.

[18] Fan, Y., Jiang, W. and Zou, Y. (2009) Numerical Simulation of Pulsatile Non-Newtonian Flow in the Carotid Artery Bifurcation. Acta Mechanica Sinica, 25, 249-255. https://doi.org/10.1007/s10409-009-0227-9

[19] Stelmakeiene, A., Ramanauskiene, K., Briedis, V. and Leskauskaite, D. (2012) Examination of Rheological and Physicochemical Charateristics in Lithuanian Honey. African Journal of Biotechnology, 11, 12406-12414.

[20] Cohen, I. and Wehs, D. (2010) Rheology and Microrheology of Natural and Reduced-Calorie Israeli Honeys as a Model for High-Viscosity Newtonian Liquids. Journal of Food Engineering, 100, 366-371. https://doi.org/10.1016/j.jfoodeng.2010.04.023

[21] Mossel, B., Bhandari, B., D’Arcy, B. and Caffin, N. (2000) Use of an Arhenius Model to Predict Rheological Behaviour of Some Australia Honeys. LWT-Food Science 
and Technology, 33, 545-552. https://doi.org/10.1006/fstl.2000.0714

[22] Munro, J.A. (1943) The Viscosity and Thixotropy of Honey. Journal of Economic Entomology, 36, 769-777.

[23] Zaitoun, S., Ghzawi, A., Al-Malah, K.I.M. and Abu-Jdayil, B. (2001) Rheological Properties of Selected Light Colored Jordanian Honey. International Journal of Food Properties, 4, 139-148. https://doi.org/10.1081/JFP-100002192

[24] Anidiobu, V.O. (2016) Rheological Characterization of Honey: Application as an Index of Quality. Ph.D. Thesis, University of Lagos, Nigeria.

[25] Bacr, J.C. and Perzynski, R. (1995) Negative Viscosity: Effect in a Magnetic Fluid. Physical Review Letters, 75, 2128-2131. https://doi.org/10.1103/PhysRevLett.75.2128

[26] Lemarchand, C.A., Bailey, N.P., Todd, B.D., Daivis, P.J. and Hansen, J.S. (2015) Non-Newtonian Behaviour and Molecular Structure of Cooee Bitumen under Shear Flow: A Non-Equilibrium Molecular Dynamic Study.

https://aip.scitation.org/doi/abs/10.1063/1.4922831 